\title{
A Constructive Scheme for a Common Coupled Fixed Point Problems in Hilbert Space
}

\author{
Kyung Soo Kim 1 \\ Graduate School of Education, Mathematics Education, Kyungnam University, Changwon 51767, Korea; \\ kksmj@kyungnam.ac.kr
}

Received: 13 September 2020; Accepted: 24 September 2020; Published: 7 October 2020

\begin{abstract}
Coupled fixed points have become the focus of interest in recent times, especially for their potential applications. Very recently, the idea of common coupled fixed point iterations has been introduced for approximating common coupled fixed points in linear spaces. Here, a coupled Mann pair iterative scheme is defined and is applied to the problem of finding common coupled fixed points of certain mappings. The discussion of the paper is in the context of Hilbert spaces.
\end{abstract}

Keywords: common coupled fixed point; Condition A; Condition B; coupled Mann pair iterative scheme; converge; Hilbert space

MSC: 47H10; 54H25

\section{Introduction}

In recent times, one of the most attractive research topics in fixed point theory is to prove the existence of a fixed point on metric spaces endowed with partial orders [1,2]. The existence of a fixed point for contraction-type mappings in partially ordered metric spaces has been considered recently by Ran et al. [2], Bhaskar et al. [3], Lakshmikantham et al. [4], and Luong et al. [5]. After the appearance of the first works in this sense, the literature on this topic has expanded significantly [2-4].

One of the reasons for such extensive interest in this category of problems is their potentiality of applications. An application to boundary value problems appeared in the work of Bhaskar et al. [3].

In [3,5], the authors proved some coupled fixed point theorems and noted their results can be used to investigate a large class of problems and have discussed the existence and uniqueness of solution for a periodic boundary value problem and a nonlinear integral equation.

In order to guarantee the existence and uniqueness of a solution of periodic boundary value problems, Bhaskar et al. [3] proved, in 2006, the existence and uniqueness of a coupled fixed point in the setting of partially ordered metric spaces $[4,6]$.

Many results were proved to ensure the existence of a coupled fixed point. One of the common properties of all these results is the fact that the mapping $F: X \times X \rightarrow X$ must verify the mixed monotone property.

Bhaskar et al. [3] introduced the notion of mixed monotone property, which has been one of the most usual hypotheses in this kind of results.

Definition 1 ([3]). Let $(X, \leq)$ be a partially ordered set and $F: X \times X \rightarrow X$. We say that $F$ has the mixed monotone property if $F(x, y)$ is monotone nondecreasing in $x$ and is monotone nonincreasing in $y$, that is, for any $x, y \in X$,

$$
\begin{array}{ll}
x_{1}, x_{2} \in X, & x_{1} \leq x_{2} \Rightarrow F\left(x_{1}, y\right) \leq F\left(x_{2}, y\right) \\
y_{1}, y_{2} \in X, & y_{1} \leq y_{2} \Rightarrow F\left(x, y_{1}\right) \leq F\left(x, y_{2}\right)
\end{array}
$$


Definition 2. For any nonempty set $X$ and a mapping $F: X \times X \rightarrow X,(x, y) \in X \times X$ is said to be a coupled fixed point of $F$, if

$$
F(x, y)=x \quad \text { and } \quad F(y, x)=y
$$

Some instances of the works following the work of Bhaskar et al. [3] are noted in [4,7-11]. However, some theorems avoiding these conditions have appeared very recently (see, for instance, [12]). Different extensions to coupled, tripled, quadrupled and multidimensional cases have appeared [13].

The Mann iteration [14] is the earliest known iteration discussed in linear spaces except the most widely used Picard iteration. Some most recent references on Mann iteration process are [15-18]. The Mann iteration is as follows:

Let $C$ be a closed convex subset of a Hilbert space $H$ and $T: C \rightarrow C$ be a self-mapping. Then for $x_{0} \in C$,

$$
x_{n+1}=\left(1-\alpha_{n}\right) x_{n}+\alpha_{n} T x_{n}, \quad n \geq 0 .
$$

where $\left\{\alpha_{n}\right\} \subset(0,1)$ satisfy certain suitable control conditions.

In a recent paper [19], the authors have initiated the study of coupled fixed point iterations by introducing a coupled Mann iterative scheme and applied the same to approximate coupled fixed points of certain mappings in the context of Hilbert spaces.

Condition A. Let $H$ be a Hilbert space and $C$ be a nonempty closed convex subset of $H$. Let $F$ : $C \times C \rightarrow C$ be any mapping. $F$ is said to satisfy Condition $A$ if for all $x, y, p, q \in C$ such that

$$
\begin{aligned}
& \|F(x, y)-F(p, q)\|^{2}+\|F(y, x)-F(q, p)\|^{2} \\
& \leq a\left[\|x-p\|^{2}+\|y-q\|^{2}\right] \\
& \quad+b\left[\left(\|p-F(p, q)\|^{2}+\|q-F(q, p)\|^{2}\right)\left(1+\|x-F(x, y)\|^{2}+\|y-F(y, x)\|^{2}\right)\right. \\
& \left.\quad+\left(\|x-F(x, y)\|^{2}+\|y-F(y, x)\|^{2}\right)\left(1+\|p-F(p, q)\|^{2}+\|q-F(q, p)\|^{2}\right)\right],
\end{aligned}
$$

where $a, b>0$ and $b<\frac{1}{4}$.

A similar condition in the corresponding uncoupled case has been considered in [20].

Coupled Mann iterative scheme. Let $H$ be a Hilbert space and $C$ be a nonempty closed convex subset of $H$. Let $F: C \times C \rightarrow C$ be any mapping. Let $\left\{x_{n}\right\}$ and $\left\{y_{n}\right\}$ be sequences in $C$ iteratively defined as follows:

$$
\begin{gathered}
\left\{\begin{array}{l}
x_{n+1}=\left(1-\alpha_{n}\right) x_{n}+\alpha_{n} F\left(x_{n}, y_{n}\right), \\
y_{n+1}=\left(1-\alpha_{n}\right) y_{n}+\alpha_{n} F\left(y_{n}, x_{n}\right), \quad n \geq 0,
\end{array}\right. \\
0<\alpha_{n}<1, \quad n=0,1,2, \cdots, \\
\quad 0<\lim _{n \rightarrow \infty} \alpha_{n}=d<1 .
\end{gathered}
$$

Theorem 1 ([19]). Let $F: C \times C \rightarrow C$ be any mapping defined on a closed nonempty convex subset $C$ of a Hilbert space $H$ such that F satisfies Condition A. Then, the coupled Mann iteration scheme constructed in (2)-(4) with $d$ in (4) satisfying $\frac{7}{4(2-b)}<d<1$, if convergent, converges to a coupled fixed point of $F$.

Coupled fixed points have become the focus of interest in recent times, especially for their potential applications. Very recently, the idea of common coupled fixed point iterations has been introduced for approximating common coupled fixed points in linear spaces. Our purpose in this paper is to introduce a new algorithm which is called the coupled Mann pair iterative scheme for arriving at a common coupled fixed point for certain mappings. The discussion of the paper is in the context of Hilbert spaces. There is a vast literature in fixed point theory on fixed point iterations. 


\section{Preliminaries}

Definition 3. For any nonempty set $X$ and mappings $S, T: X \times X \rightarrow X,(x, y) \in X \times X$ is said to be a common coupled fixed point of $S$ and $T$, if

$$
S(x, y)=x, \quad S(y, x)=y, \quad T(x, y)=x, \quad T(y, x)=y .
$$

The following are some technical descriptions which we require in this paper. We use the following contractive inequality condition on $S$ and $T$ which we write as Condition A and Condition B.

Definition 4. Let $H$ be a Hilbert space and $C$ be a nonempty closed convex subset of $H$. Let $S, T: C \times C \rightarrow C$ be any mappings.

(1) $(S, T)$ is said to satisfy Condition $A$ if for all $x, y, p, q \in C$,

$$
\begin{aligned}
& \|S(x, y)-S(p, q)\|^{2}+\|T(y, x)-T(q, p)\|^{2} \\
& \leq a\left(\|x-p\|^{2}+\|y-q\|^{2}\right) \\
& \quad+b\left\{\left(\|p-S(p, q)\|^{2}+\|q-T(q, p)\|^{2}\right)\left(1+\|x-S(x, y)\|^{2}+\|y-T(y, x)\|^{2}\right)\right. \\
& \left.\quad+\left(\|x-S(x, y)\|^{2}+\|y-T(y, x)\|^{2}\right)\left(1+\|p-S(p, q)\|^{2}+\|q-T(q, p)\|^{2}\right)\right\},
\end{aligned}
$$

(2) $(S, T)$ is said to satisfy Condition B if for all $x, y, p, q \in C$,

$$
\begin{aligned}
& \|T(x, y)-S(p, q)\|^{2}+\|S(y, x)-T(q, p)\|^{2} \\
& \leq a\left(\|x-p\|^{2}+\|y-q\|^{2}\right) \\
& \quad+b\left\{\left(\|p-S(p, q)\|^{2}+\|q-T(q, p)\|^{2}\right)\left(1+\|x-T(x, y)\|^{2}+\|y-S(y, x)\|^{2}\right)\right. \\
& \left.\quad+\left(\|x-T(x, y)\|^{2}+\|y-S(y, x)\|^{2}\right)\left(1+\|p-S(p, q)\|^{2}+\|q-T(q, p)\|^{2}\right)\right\},
\end{aligned}
$$

where $a, b>0$ and $b<\frac{1}{4}$.

Here, we introduce a coupled Mann pair iteration scheme.

Coupled Mann pair iterative scheme: Let $H$ be a Hilbert space and $C$ be a nonempty closed convex subset of $H$. Let $S, T: C \times C \rightarrow C$ be any mappings. Let $\left\{x_{n}\right\}$ and $\left\{y_{n}\right\}$ be sequences in $C$ iteratively defined as follows:

$$
\left\{\begin{array}{l}
x_{n+1}=\left(1-\alpha_{n}\right) x_{n}+\alpha_{n} S\left(x_{n}, y_{n}\right), \\
y_{n+1}=\left(1-\alpha_{n}\right) y_{n}+\alpha_{n} T\left(y_{n}, x_{n}\right), \quad n \geq 0
\end{array}\right.
$$

where

$$
\begin{aligned}
& 0<\alpha_{n}<1, \quad n=0,1,2, \cdots, \\
& 0<\lim _{n \rightarrow \infty} \alpha_{n}=d<1 .
\end{aligned}
$$

\section{Main Results}

Theorem 2. Let $S, T: C \times C \rightarrow C$ be any mappings defined on a closed nonempty convex subset $C$ of a Hilbert space $H$ such that $(S, T)$ satisfies Condition A and Condition B. Then the coupled Mann pair iterative scheme constructed in (5)-(7) with $d$ in (7) satisfying $\frac{7}{4(2-b)}<d<1$, if convergent, converges to a common coupled fixed point of $S$ and $T$.

Proof. Let $\left(x_{n}, y_{n}\right) \rightarrow(x, y)$ as $n \rightarrow \infty$. 
I. Using parallelogram law, we get

$$
\begin{aligned}
& \|S(x, y)-x\|^{2}+\|T(y, x)-y\|^{2} \\
& =\left\|S(x, y)-x_{n+1}+x_{n+1}-x\right\|^{2}+\left\|T(y, x)-y_{n+1}+y_{n+1}-y\right\|^{2} \\
& \leq 2\left\|S(x, y)-x_{n+1}\right\|^{2}+2\left\|x_{n+1}-x\right\|^{2}+2\left\|T(y, x)-y_{n+1}\right\|^{2}+2\left\|y_{n+1}-y\right\|^{2},
\end{aligned}
$$

for all $x, y \in C$. Since

$$
\begin{aligned}
\left\|S(x, y)-x_{n+1}\right\|^{2} & =\left\|\left(1-\alpha_{n}\right)\left(S(x, y)-x_{n}\right)+\alpha_{n}\left(S(x, y)-S\left(x_{n}, y_{n}\right)\right)\right\|^{2} \\
& \leq 2\left(1-\alpha_{n}\right)^{2}\left\|S(x, y)-x_{n}\right\|^{2}+2 \alpha_{n}^{2}\left\|S(x, y)-S\left(x_{n}, y_{n}\right)\right\|^{2}
\end{aligned}
$$

and

$$
\begin{aligned}
\left\|T(y, x)-y_{n+1}\right\|^{2} & =\left\|\left(1-\alpha_{n}\right)\left(T(y, x)-y_{n}\right)+\alpha_{n}\left(T(y, x)-T\left(y_{n}, x_{n}\right)\right)\right\|^{2} \\
& \leq 2\left(1-\alpha_{n}\right)^{2}\left\|T(y, x)-y_{n}\right\|^{2}+2 \alpha_{n}^{2}\left\|T(y, x)-T\left(y_{n}, x_{n}\right)\right\|^{2},
\end{aligned}
$$

using Condition A, (9) and (10) in (8), we get

$$
\begin{aligned}
\| & S(x, y)-x\left\|^{2}+\right\| T(y, x)-y \|^{2} \\
\leq & 2\left\|x_{n+1}-x\right\|^{2}+2\left\|y_{n+1}-y\right\|^{2} \\
& +4\left(1-\alpha_{n}\right)^{2}\left\|S(x, y)-x_{n}\right\|^{2}+4 \alpha_{n}^{2}\left\|S(x, y)-S\left(x_{n}, y_{n}\right)\right\|^{2} \\
& +4\left(1-\alpha_{n}\right)^{2}\left\|T(y, x)-y_{n}\right\|^{2}+4 \alpha_{n}^{2}\left\|T(y, x)-T\left(y_{n}, x_{n}\right)\right\|^{2} \\
\leq & 2\left\|x_{n+1}-x\right\|^{2}+2\left\|y_{n+1}-y\right\|^{2} \\
& +4 \alpha_{n}^{2}\left(\left\|S(x, y)-S\left(x_{n}, y_{n}\right)\right\|^{2}+\left\|T(y, x)-T\left(y_{n}, x_{n}\right)\right\|^{2}\right) \\
& +4\left(1-\alpha_{n}\right)^{2}\left[2\|S(x, y)-x\|^{2}+2\left\|x-x_{n}\right\|^{2}\right]+4\left(1-\alpha_{n}\right)^{2}\left[2\|T(y, x)-y\|^{2}+2\left\|y-y_{n}\right\|^{2}\right] \\
\leq & 2\left\|x_{n+1}-x\right\|^{2}+2\left\|y_{n+1}-y\right\|^{2}+4 \alpha_{n}^{2}\left[a\left(\left\|x-x_{n}\right\|^{2}+\left\|y-y_{n}\right\|^{2}\right)\right. \\
& +b\left\{\left(\left\|x_{n}-S\left(x_{n}, y_{n}\right)\right\|^{2}+\left\|y_{n}-T\left(y_{n}, x_{n}\right)\right\|^{2}\right)\left(1+\|x-S(x, y)\|^{2}+\|y-T(y, x)\|^{2}\right)\right. \\
& \left.\left.+\left(\|x-S(x, y)\|^{2}+\|y-T(y, x)\|^{2}\right)\left(1+\left\|x_{n}-S\left(x_{n}, y_{n}\right)\right\|^{2}+\left\|y_{n}-T\left(y_{n}, x_{n}\right)\right\|^{2}\right)\right\}\right] \\
& +8\left(1-\alpha_{n}\right)^{2}\left[\|S(x, y)-x\|^{2}+\|T(y, x)-y\|^{2}+\left\|x-x_{n}\right\|^{2}+\left\|y-y_{n}\right\|^{2}\right] .
\end{aligned}
$$

Since

$$
\left\|x_{n}-S\left(x_{n}, y_{n}\right)\right\|^{2}=\frac{1}{\alpha_{n}^{2}}\left\|x_{n}-x_{n+1}\right\|^{2}
$$

and

$$
\left\|y_{n}-T\left(y_{n}, x_{n}\right)\right\|^{2}=\frac{1}{\alpha_{n}^{2}}\left\|y_{n}-y_{n+1}\right\|^{2},
$$


(11) have

$$
\begin{aligned}
& \|S(x, y)-x\|^{2}+\|T(y, x)-y\|^{2} \\
& \leq 2\left\|x_{n+1}-x\right\|^{2}+2\left\|y_{n+1}-y\right\|^{2} \\
& \quad+8\left(1-\alpha_{n}\right)^{2}\left[\|S(x, y)-x\|^{2}+\|T(y, x)-y\|^{2}+\left\|x-x_{n}\right\|^{2}+\left\|y-y_{n}\right\|^{2}\right] \\
& \quad+4 \alpha_{n}^{2}\left[a\left(\left\|x-x_{n}\right\|^{2}+\left\|y-y_{n}\right\|^{2}\right)\right. \\
& \quad+b\left\{\frac{1}{\alpha_{n}^{2}}\left(\left\|x_{n}-x_{n+1}\right\|^{2}+\left\|y_{n}-y_{n+1}\right\|^{2}\right)\left(1+\|x-S(x, y)\|^{2}+\|y-T(y, x)\|^{2}\right)\right. \\
& \left.\left.\quad+\left(\|x-S(x, y)\|^{2}+\|y-T(y, x)\|^{2}\right)\left(1+\frac{1}{\alpha_{n}^{2}}\left(\left\|x_{n}-x_{n+1}\right\|^{2}+\left\|y_{n}-y_{n+1}\right\|^{2}\right)\right)\right\}\right] .
\end{aligned}
$$

Taking $n \rightarrow \infty$ in (14), by (7), we obtain

$$
\begin{aligned}
\|S(x, y)-x\|^{2}+\|T(y, x)-y\|^{2} \leq & 8(1-d)^{2}\left[\|S(x, y)-x\|^{2}+\|T(y, x)-y\|^{2}\right] \\
& +4 d^{2} b\left\{\|x-S(x, y)\|^{2}+\|y-T(y, x)\|^{2}\right\} \\
= & 4\left(2(1-d)^{2}+b d^{2}\right)\left(\|S(x, y)-x\|^{2}+\|T(y, x)-y\|^{2}\right) .
\end{aligned}
$$

From $b<\frac{1}{4}$ and $0<d<1$, we obtain

$$
\begin{aligned}
2(1-d)^{2}+b d^{2} & =2-4 d+2 d^{2}+b d^{2} \\
& \leq 2-4 d+2 d+b d \\
& =2-(2-b) d
\end{aligned}
$$

Since $\frac{7}{4(2-b)}<d$, we get

$$
2(1-d)^{2}+b d^{2}<\frac{1}{4}
$$

From (15) and (16), we have

$$
\|S(x, y)-x\|^{2}+\|T(y, x)-y\|^{2}=0 .
$$

Hence,

$$
\|S(x, y)-x\|=0 \quad \text { and } \quad\|T(y, x)-y\|=0 .
$$

Therefore,

$$
S(x, y)=x \quad \text { and } \quad T(y, x)=y
$$

II. Using parallelogram law, we obtain

$$
\begin{aligned}
& \|T(x, y)-x\|^{2}+\|S(y, x)-y\|^{2} \\
& =\left\|T(x, y)-x_{n+1}+x_{n+1}-x\right\|^{2}+\left\|S(y, x)-y_{n+1}+y_{n+1}-y\right\|^{2} \\
& \leq 2\left\|T(x, y)-x_{n+1}\right\|^{2}+2\left\|x_{n+1}-x\right\|^{2}+2\left\|S(y, x)-y_{n+1}\right\|^{2}+2\left\|y_{n+1}-y\right\|^{2},
\end{aligned}
$$

for all $x, y \in C$. Since

$$
\begin{aligned}
\left\|T(x, y)-x_{n+1}\right\|^{2} & =\left\|\left(1-\alpha_{n}\right)\left(T(x, y)-x_{n}\right)+\alpha_{n}\left(T(x, y)-S\left(x_{n}, y_{n}\right)\right)\right\|^{2} \\
& \leq 2\left(1-\alpha_{n}\right)^{2}\left\|T(x, y)-x_{n}\right\|^{2}+2 \alpha_{n}^{2}\left\|T(x, y)-S\left(x_{n}, y_{n}\right)\right\|^{2}
\end{aligned}
$$


and

$$
\begin{aligned}
\left\|S(y, x)-y_{n+1}\right\|^{2} & =\left\|\left(1-\alpha_{n}\right)\left(S(y, x)-y_{n}\right)+\alpha_{n}\left(S(y, x)-T\left(y_{n}, x_{n}\right)\right)\right\|^{2} \\
& \leq 2\left(1-\alpha_{n}\right)^{2}\left\|S(y, x)-y_{n}\right\|^{2}+2 \alpha_{n}^{2}\left\|S(y, x)-T\left(y_{n}, x_{n}\right)\right\|^{2},
\end{aligned}
$$

using Condition B, (18) and (19) in (17), we have

$$
\begin{aligned}
\| & T(x, y)-x\left\|^{2}+\right\| S(y, x)-y \|^{2} \\
\leq & 2\left\|x_{n+1}-x\right\|^{2}+2\left\|y_{n+1}-y\right\|^{2} \\
& +4\left(1-\alpha_{n}\right)^{2}\left\|T(x, y)-x_{n}\right\|^{2}+4 \alpha_{n}^{2}\left\|T(x, y)-S\left(x_{n}, y_{n}\right)\right\|^{2} \\
& +4\left(1-\alpha_{n}\right)^{2}\left\|S(y, x)-y_{n}\right\|^{2}+4 \alpha_{n}^{2}\left\|S(y, x)-T\left(y_{n}, x_{n}\right)\right\|^{2} \\
\leq & 2\left\|x_{n+1}-x\right\|^{2}+2\left\|y_{n+1}-y\right\|^{2} \\
& +4\left(1-\alpha_{n}\right)^{2}\left(2\|T(x, y)-x\|^{2}+2\left\|x-x_{n}\right\|^{2}\right) \\
& +4\left(1-\alpha_{n}\right)^{2}\left(2\|S(y, x)-y\|^{2}+2\left\|y-y_{n}\right\|^{2}\right) \\
& +4 \alpha_{n}^{2}\left(\left\|T(x, y)-S\left(x_{n}, y_{n}\right)\right\|^{2}+\left\|S(y, x)-T\left(y_{n}, x_{n}\right)\right\|^{2}\right) \\
\leq & 2\left\|x_{n+1}-x\right\|^{2}+2\left\|y_{n+1}-y\right\|^{2} \\
& +8\left(1-\alpha_{n}\right)^{2}\left[\|T(x, y)-x\|^{2}+\|S(y, x)-y\|^{2}+\left\|x-x_{n}\right\|^{2}+\left\|y-y_{n}\right\|^{2}\right] \\
& +4 \alpha_{n}^{2}\left[a\left(\left\|x-x_{n}\right\|^{2}+\left\|y-y_{n}\right\|^{2}\right)\right. \\
& +b\left\{\left(\left\|x_{n}-S\left(x_{n}, y_{n}\right)\right\|^{2}+\left\|y_{n}-T\left(y_{n}, x_{n}\right)\right\|^{2}\right)\left(1+\|x-T(x, y)\|^{2}+\|y-S(y, x)\|^{2}\right)\right. \\
& \left.\left.+\left(\|x-T(x, y)\|^{2}+\|y-S(y, x)\|^{2}\right)\left(1+\left\|x_{n}-S\left(x_{n}, y_{n}\right)\right\|^{2}+\left\|y_{n}-T\left(y_{n}, x_{n}\right)\right\|^{2}\right)\right\}\right] .
\end{aligned}
$$

By (12) and (13), (20) have

$$
\begin{aligned}
& \|T(x, y)-x\|^{2}+\|S(y, x)-y\|^{2} \\
& \leq \\
& \quad 2\left\|x_{n+1}-x\right\|^{2}+2\left\|y_{n+1}-y\right\|^{2} \\
& +8\left(1-\alpha_{n}\right)^{2}\left[\|T(x, y)-x\|^{2}+\|S(y, x)-y\|^{2}+\left\|x-x_{n}\right\|^{2}+\left\|y-y_{n}\right\|^{2}\right] \\
& \quad+4 \alpha_{n}^{2}\left[a\left(\left\|x-x_{n}\right\|^{2}+\left\|y-y_{n}\right\|^{2}\right)\right. \\
& \quad+b\left\{\frac{1}{\alpha_{n}^{2}}\left(\left\|x_{n}-x_{n+1}\right\|^{2}+\left\|y_{n}-y_{n+1}\right\|^{2}\right)\left(1+\|x-T(x, y)\|^{2}+\|y-S(y, x)\|^{2}\right)\right. \\
& \left.\left.\quad+\left(\|x-T(x, y)\|^{2}+\|y-S(y, x)\|^{2}\right)\left(1+\frac{1}{\alpha_{n}^{2}}\left(\left\|x_{n}-x_{n+1}\right\|^{2}+\left\|y_{n}-y_{n+1}\right\|^{2}\right)\right)\right\}\right] .
\end{aligned}
$$

Taking $n \rightarrow \infty$ in (21), by (7), we obtain

$$
\begin{aligned}
\|T(x, y)-x\|^{2}+\|S(y, x)-y\|^{2} \leq & 8(1-d)^{2}\left[\|T(x, y)-x\|^{2}+\|S(y, x)-y\|^{2}\right] \\
& +4 d^{2} b\left\{\|x-T(x, y)\|^{2}+\|y-S(y, x)\|^{2}\right\} \\
= & 4\left(2(1-d)^{2}+b d^{2}\right)\left(\|T(x, y)-x\|^{2}+\|S(y, x)-y\|^{2}\right) .
\end{aligned}
$$

Since $b<\frac{1}{4}$ and $\frac{7}{4(2-b)}<d<1$, we have

$$
2(1-d)^{2}+b d^{2}<\frac{1}{4}
$$


From (22) and (23), we get

$$
\|T(x, y)-x\|^{2}+\|S(y, x)-y\|^{2}=0 .
$$

Therefore,

$$
T(x, y)=x \quad \text { and } \quad S(y, x)=y
$$

By $\mathbf{I}$ and $\mathbf{I I},(x, y)$ is a common coupled fixed point of $S$ and $T$. This completes the proof.

Corollary 1. Let $S: C \times C \rightarrow C$ be any mapping defined on a closed nonempty convex subset $C$ of a Hilbert space $H$ such that $S$ satisfies

$$
\begin{aligned}
& \|S(x, y)-S(p, q)\|^{2}+\|S(y, x)-S(q, p)\|^{2} \\
& \leq a\left(\|x-p\|^{2}+\|y-q\|^{2}\right) \\
& \quad+b\left\{\left(\|p-S(p, q)\|^{2}+\|q-S(q, p)\|^{2}\right)\left(1+\|x-S(x, y)\|^{2}+\|y-S(y, x)\|^{2}\right)\right. \\
& \left.\quad+\left(\|x-S(x, y)\|^{2}+\|y-S(y, x)\|^{2}\right)\left(1+\|p-S(p, q)\|^{2}+\|q-S(q, p)\|^{2}\right)\right\},
\end{aligned}
$$

for all $x, y, p, q \in C$. Then the coupled Mann iterative scheme

$$
\left\{\begin{array}{l}
x_{n+1}=\left(1-\alpha_{n}\right) x_{n}+\alpha_{n} S\left(x_{n}, y_{n}\right), \\
y_{n+1}=\left(1-\alpha_{n}\right) y_{n}+\alpha_{n} S\left(y_{n}, x_{n}\right), \quad n \geq 0
\end{array}\right.
$$

where

$$
\begin{aligned}
& 0<\alpha_{n}<1, \quad n=0,1,2, \cdots, \\
& 0<\lim _{n \rightarrow \infty} \alpha_{n}=d<1
\end{aligned}
$$

constructed in (25)-(27) with $d$ in (27) satisfying $\frac{7}{4(2-b)}<d<1$, if convergent, converges to a coupled fixed point of $S$.

Proof. Taking $S=T$ in Theorem 2, then Condition A and Condition B are equal to (24) and the coupled Mann pair iterative scheme is equal to coupled Mann iterative scheme. Thus, we have the conclusion of the Corollary follows. Moreover, it becomes Theorem 1. So, Theorem 1 can be treated as a special case of Theorem 2 .

We define as $F(x)=S(x, x)$ and $G(x)=T(x, x)$. Then, we have the following Corollary.

Corollary 2. Let $F, G: C \rightarrow C$ be any mappings defined on a closed nonempty convex subset $C$ of a Hilbert space $H$ such that $F$ and $G$ satisfy

$$
\begin{aligned}
& \|F(x)-F(p)\|^{2}+\|G(x)-G(p)\|^{2} \\
& \leq a\|x-p\|^{2} \\
& \quad+b\left[\left(\|p-F(p)\|^{2}+\|p-G(p)\|^{2}\right)\left(1+\|x-F(x)\|^{2}+\|x-G(x)\|^{2}\right)\right. \\
& \left.\quad+\left(\|x-F(x)\|^{2}+\|x-G(x)\|^{2}\right)\left(1+\|p-F(p)\|^{2}+\|p-G(p)\|^{2}\right)\right], \quad \forall x, p \in C,
\end{aligned}
$$

where $a, b>0$ and $b<\frac{1}{4}$. Then the Mann pair iterative scheme

$$
\left\{\begin{array}{l}
x_{n+1}=\left(1-\alpha_{n}\right) x_{n}+\alpha_{n} F\left(x_{n}\right) \\
y_{n+1}=\left(1-\alpha_{n}\right) y_{n}+\alpha_{n} G\left(x_{n}\right), \quad n \geq 0
\end{array}\right.
$$


where

$$
\begin{aligned}
& 0<\alpha_{n}<1, \quad n=0,1,2, \cdots, \\
& 0<\lim _{n \rightarrow \infty} \alpha_{n}=d<1
\end{aligned}
$$

satisfying $\frac{7}{4(2-b)}<d<1$, if convergent, converges to a common fixed point of $F$ and $G$.

Proof. Taking $x=y$ and $p=q$ in Theorem 2, then the conclusion of the Corollary follows.

Example 1. Define $S, T: \mathbb{R} \times \mathbb{R} \rightarrow \mathbb{R}$ by

$$
S(x, y)=\left\{\begin{array}{ll}
\frac{1}{2}(x-y), & \text { if } x \geq y, \\
0, & \text { if } x<y,
\end{array} \quad T(p, q)= \begin{cases}0, & \text { if } p \geq q, \\
\frac{1}{2}(p-q), & \text { if } p<q,\end{cases}\right.
$$

where $\mathbb{R}$ is the set of real numbers. Clearly $(0,0)$ is the common coupled fixed point of $S$ and $T$. $(S, T)$ satisfies Condition $A$ and Condition $B$ for $a>1$ and $b>0$.

Funding: This research was supported by Basic Science Research Program through the National Research Foundation of Korea (NRF) funded by the Ministry of Education (2019R1I1A3A01060344).

Acknowledgments: The author would like to thank the referees for their valuable comments and suggestions which improved the presentation of this paper.

Conflicts of Interest: The author declares that they have no competing interest.

\section{References}

1. Turinici, M. Abstract comparison principles and multivariable Gronwall-Bellman inequalities. J. Math. Anal. Appl. 1986, 117, 100-127. [CrossRef]

2. Ran, A.C.M.; Reurings, M.C.B. A fixed point theorem in partially ordered sets and some applications to matrix equations. Proc. Am. Math. Soc. 2004, 132, 1435-1443. [CrossRef]

3. Bhaskar, T.G.; Lakshmikantham, V. Fixed point theorems in partially ordered metric spaces and applications. Nonlinear Anal. 2006, 65, 1379-1393. [CrossRef]

4. Lakshmikantham, V.; Ciric, L. Coupled fixed point theorems for non linear contractions in partially ordered metric spaces. Nonlinear Anal. 2009, 70, 4341-4349. [CrossRef]

5. Luong, N.V.; Thuan, N.X. Coupled fixed points in partially ordered metric spaces and application. Nonlinear Anal. 2011, 74, 983-992. [CrossRef]

6. Guo, D.; Lakshmikantham, V. Coupled fixed points of nonlinear operators with applications. Nonlinear Anal. 1987, 11, 623-632. [CrossRef]

7. Choudhury, B.S.; Maity, P. Coupled fixed points results in generalized metric spaces. Math. Comput. Model. 2011, 54, 73-79. [CrossRef]

8. Ding, H.S.; Karapinar, E. A note on some coupled fixed point theorems on G-metric spaces. J. Inequal. Appl. 2012, 2012, 170. [CrossRef]

9. Rezaee, M.M.; Sedghi, S.; Kim, K.S. Coupled common fixed point results in ordered S-metric spaces. Nonlinear Funct. Anal. Appl. 2018, 23, 595-612.

10. Roldán-López-de-Hierro, A.F.; Karapinar, E.; de la Sen, M.D. Coincidence point theorems in quasi-metric spaces assuming the mixed monotone property and consequences in G-metric spaces. Fixed Point Theory Appl. 2014, 2014, 184. [CrossRef]

11. Singh, S.L.; Kamal, R.; Sen, M.D. Coincidence and common fixed point theorems for Suzuki type hybrid contractions and applications. Fixed Point Theory Appl. 2014, 2014, 147. [CrossRef]

12. Kutbi, M.A.; Roldán, A.; Sintunavarat, W.; Martínez-Moreno, J.; Roldán, C. F-Closed sets and coupled fixed point theorems without the mixed monotone property. Fixed Point Theory Appl. 2013, 2013, 330. [CrossRef]

13. Amini-Harandi, A. Coupled and tripled fixed point theory in partially ordered metric spaces with application to initial value theorem. Math. Comput. Model. 2013, 57, 2343-2348. [CrossRef] 
14. Mann, W.R. Mean value methods in iteration. Proc. Am. Math. Soc. 1953, 4, 506-510. [CrossRef]

15. Dehaish, B.A.I.; Khamsi, M.A.; Khan, A.R. Mann iteration process for asymptotic pointwise nonexpansive mappings in metric spaces. J. Math. Anal. Appl. 2013, 397, 861-868. [CrossRef]

16. Kim, K.S. Convergence theorems of variational inequality for asymptotically nonexpansive nonself mapping in CAT(0) spaces. Mathematics 2019, 7, 1234. [CrossRef]

17. Maruster, L.; Maruster, S. Strong convergence of the Mann iteration for $\alpha$-demicontractive mappings. Math. Comput. Model. 2011, 54, 2486-2492. [CrossRef]

18. Zegeye, H.; Shahzad, N. Convergence of Mann's type iteration method for generalized asymptotically nonexpansive mappings. Comput. Math. Appl. 2011, 62, 4007-4014. [CrossRef]

19. Choudhury, B.S.; Kundu, S. A constructive algorithm for a coupled fixed point problem. Nonlinear Funct. Anal. Appl. 2016, 21, 567-572.

20. Choudhury, B.S. Random Mann iteration scheme. Appl. Math. Lett. 2003, 16, 93-96. [CrossRef]

(C) 2020 by the author. Licensee MDPI, Basel, Switzerland. This article is an open access article distributed under the terms and conditions of the Creative Commons Attribution (CC BY) license (http:/ / creativecommons.org/licenses/by/4.0/). 